\title{
LA PRODUCTIVITÉ DE L'ÉPICÉA COMMUN SUR LES SOLS HYDROMORPHES DE LORRAINE : MESURE ET CARACTÉRISTIQUES GÉNÉRALES
}

\author{
N. DECOURT et G. LÉVY \\ Station de Sylviculture et Production, \\ Station de Recherches sur les Sols forestiers et la Fertilisation, \\ Centre national de Recherches forestières, I. N. R. A., \\ Champenoux, 54370 Einville
}

\section{RÉSUMÉ}

Une centaine de placettes a été retenue pour cette enquête sur Epicéa adulte. Des sondages à la tarière de Pressler à différentes hauteurs sur des arbres dominants ont permis de reconstituer la courbe de croissance en hauteur en fonction de l'âge puis, par inter - ou extrapolation, d'obtenir la hauteur dominante à 50 ans (indice de productivité). Cette méthode, peu précise mais rapide, convient bien à des études de liaison «station-production » sur un grand nombre de placettes.

La table de production pour l'Epicéa commun dans le Nord-Est (DecourT, 1972) s'applique bien, globalement, au cas particulier des peuplements sur sol hydromorphe, bien que dans les moins bonnes placettes 1'allure de la croissance tende à être moins rapide que ne le prévoit la table.

Seulement $8 \%$ des peuplements sont inférieurs à la classe $4:$ les facteurs limitants de ces sols hydromorphes n'affectent fortement la production que dans les cas les plus défavorables.

\section{1. - INTRODUCTION}

Nous avons procédé à une «enquête " sol - nutrition - production (GARBAYE et al., 1970) pour l'Épicéa sur les sols lourds, en particulier les sols à hydromorphie temporaire de Lorraine. Il était nécessaire de déterminer, pour chaque placette, un indice de productivité : la hauteur dominante à un âge de référence. La table de production de l'Épicéa, pour le Nord-Est (DeCOURT, 1972), permet d'obtenir cet indice à partir de la hauteur dominante de la placette au moment de la mesure. Mais cette table ne fournit qu'une valeur moyenne, qui est peut-être assez éloignée de la valeur réelle dans le cas de certaines placettes; de plus, parmi les placettes utilisées pour construire la table, peu correspondaient à des sols lourds.

La méthode classique d'évaluation de la hauteur dominante à un âge donné consiste à effectuer des analyses de tiges d'arbres dominants. Malheureusement cette méthode est 
longue et coûteuse. Nous avons donc mis au point une méthode plus rapide, mais sans doute également moins précise. Le présent article se propose trois objectifs :

- Comparer cette méthode simplifiée avec celle fondée sur l'analyse de tiges.

- Examiner les conditions d'application de la table de production pour l'Épicéa commun dans le Nord-Est de la France, dans le cas particulier des sols à hydromorphie temporaire :

En effet, les sols étudiés au cours de cette enquête présentent un facteur sans doute défavorable à la croissance des arbres : la présence d'une nappe d'eau temporaire (ou, pour le moins, l'existence d'un niveau très argileux à propriétés intrinsèques défavorables). A priori, il n'est pas impossible que ce facteur modifie l'allure de la courbe de croissance en fonction de l'âge, par rapport à celle de la table de production, et celà pour une même classe de production. Ainsi, par exemple, il y a peut-être un freinage assez brutal de la croissance au moment où les racines du peuplement ayant colonisé toute l'épaisseur du sol sain, se heurtent à l'obstacle constitué par la nappe (ou l'argile).

- Enfin, examiner la gamme des productions que l'on obtient sur les sols étudiés. Nous saurons ainsi dans quelle mesure l'hydromorphie constitue un facteur limitant pour la croissance de l'Épicéa.

\section{2. - MATÉRIEL ET MÉTHODES}

\section{1. - Les peuplements}

La zone retenue est climatiquement assez homogène malgré, bien sûr, quelques variations; ainsi la pluviométrie varie de 700 à $1000 \mathrm{~mm}$.

Les placettes sont situées sur le Plateau lorrain, dans les départements de Moselle, Meurthe-etMoselle, et Vosges, sur roche-mère lourde (marnes ou argiles du Grès bigarré, Muschelkalk, Keuper, Sinémurien, Lotharingien, Charmouthien), très souvent surmontée d'une couche de limon d'origine probablement éolienne. Exceptionnellement, le substratum est moins argileux : il s'agit alors d'alluvions anciennes, plus ou moins recouvertes de limons, et ayant donné naissance à un pseudogley. Les sols sont variés : sols bruns marmorisés et à pseudogley, pseudogleys, pélosols-pseudogleys, pélosols; toute la gamme d'intensités et de profondeurs d'apparition de signes d'hydromorphie y est représentée. A ces traces d'hydromorphie correspond, dans la majorité des cas, une nappe temporaire de profondeur et de durée variées; mais dans un certain nombre de placettes, nous n'avons pû mettre en évidence l'existence d'une nappe; par contre, dans quelques cas, il existe une nappe permanente. Cependant, nous considérerons dans cet article que l'ensemble de nos placettes sont situées sur des sols que nous appellerons « hydromorphes ».

Les conditions topographiques sont également variées.

Les placettes, au nombre de 97 , constituent un échantillonnage représentatif des peuplements d'Epicéa sur les sols lourds de la région considérée. On ne peut cependant être certain que chaque type de sol ou d'hydromorphie soit représenté proportionnellement à sa fréquence réelle. L'âge au moment de la mesure était compris entre 36 et 69 ans.

\section{2. - L'indice de productivité}

Selon la loi de Eichhorn élargie, la production en volume depuis l'origine est fonction de la seule hauteur, du moins dans la gamme des densités de peuplement utilisées couramment.

En particulier la hauteur moyenne des arbres dominants, appelée hauteur dominante, ne dépend pas de la densité du peuplement; elle n'est fonction que de la station et bien liée à la productivité de cette dernière. 
En chaque point retenu, nous avons délimité des microplacettes (DECOURT et LE TACON, 1970) (comprenant 50 à 70 arbres), à sol homogène, puis mesuré la hauteur dominante (hauteur moyenne des arbres les plus gros à $1,30 \mathrm{~m}$, en nombre égal à $10 \%$ de l'ensemble des arbres de la micro-placette). Pour obtenir la hauteur dominante à un même âge de référence (Indice de productivité) on a cherché à reconstituer la croissance en hauteur d'un arbre dominant dans chaque placette. On a choisi pour cela, l'arbre dont la hauteur était la plus voisine de la hauteur dominante évaluée pour le peuplement.

La méthode consiste à faire des sondages à la tarière de Pressler, jusqu'au cœur, à environ 1/3 et 2/3 de la hauteur de l'arbre étudié. Par comptage des cernes annuels on évalue les âges auxquels ces hauteurs ont été atteintes. On possède ainsi 4 points connus (origine, les deux sondages, hauteur actuelle) pour reconstituer approximativement la courbe de croissance en hauteur de l'arbre considéré. Si on suppose qu'il a toujours conservé une hauteur voisine de la hauteur dominante du peuplement, on pourra à l'aide de cette courbe évaluer par interpolation ou par extrapolation la hauteur dominante probable du peuplement à un âge de référence choisi. Cette méthode est bien plus rapide et moins onéreuse que l'analyse de tiges, de plus elle n'est pas destructrive; mais les mesures peuvent être parfois assez dangereuses à pratiquer par grimpage, et sa précision laisse sans doute à désirer; nous examinerons dans quelle mesure.

\section{3. - CHOIX DE L'ÂGE DE RÉFÉRENCE ET VALEUR DE LA MÉTHODE DES SONDAGES}

\section{1. - Choix de l'âge de référence}

Plus cet âge de référence est grand, plus l'indice de productivité obtenu est effectivement en rapport avec la production totale, même si le classement relatif des peuplements a varié avec l'âge.

Si l'âge de référence est trop élevé par rapport à l'âge actuel, l'évaluation de l'indice se fera par extrapolation et donc avec une plus grande incertitude.

L'âge des placettes étudiées variant de 36 à 69 ans, nous avons néanmoins choisi de retenir comme indice de productivité la hauteur dominante à 50 ans (HD 50) à la suite des remarques exposées ci-dessous.

\section{2. - Valeur de la méthode des sondages à la tarière pour obtenir HD 50}

Nous avons comparé les résultats fournis par cette méthode avec ceux obtenus à l'aide de l'analyse complète des tiges dominantes. Dans ce but, nous avons choisi au hasard 11 courbes fournies par l'analyse de tiges parmi l'ensemble des courbes ayant servi à l'élaboration de la table de production (les peuplements correspondant ont plus de 50 ans).

\subsection{Extrapolation}

De chacune de ces 11 courbes nous avons retenu 3 points correspondant respectivement à la hauteur à 33 ans, au 2/3, et au $1 / 3$ de cette hauteur. Nous nous plaçons ainsi dans un cas très défavorable (les peuplements les plus jeunes retenus sur sol hydromorphe ont 36 ans). Nous avons tenté de tracer à l'aide de ces trois points (plus le point origine) la courbe de croissance en fonction de l'âge, de la même façon que nous l'avions fait pour les microplacettes sur sols hydromorphes, et d'en déduire HD 50 ainsi retrouvée « théoriquement". 
Nous pouvons alors comparer (Tableau 1) HD 50 et HD 50 extrapolé par notre méthode simplifiée, à partir de données ayant servi à la construction de la table.

TABLEAU 1

Comparaison de HD 50 réel

et HD 50 obtenu par la méthode des carottages pour un peuplement de 33 ans

TABLE 1

Comparison beetween real HD 50 (dominant height at 50 years) and HD 50 obtained by the boring method for a 33 years old stand

\begin{tabular}{c|c|c|c|c|c|c|c|c|c|c|c}
\hline \hline \multicolumn{1}{c|}{ Placette } & 54.18 & 52,08 & 54.19 & 54.20 & 52.07 & 52.06 & 52.05 & 21.5 & 21.3 & 21.2 & 21.1 \\
\hline $\begin{array}{l}\text { HD 50 réel (ana- } \\
\text { lyse de tige) (en } \\
\text { m). }\end{array}$ & 18,9 & 20,1 & 26,1 & 26,6 & 23,6 & 24,5 & 27,0 & 20,8 & 23,0 & 24,4 & 27,0 \\
\hline $\begin{array}{l}\text { HD 50 extrapolé } \\
\text { (en m). }\end{array}$ & 18,2 & 19,8 & 28,4 & 27,2 & 19,8 & 22,0 & 26,2 & 17,2 & 24,3 & 26,2 & 25,7 \\
\hline \hline
\end{tabular}

Pour 6 placettes sur les 11, la différence par rapport à HD 50 réel est inférieure ou égale à $1 / 2$ classe de production; pour 3 placettes, elle est comprise entre $1 / 2$ et 1 classe (les 2 valeurs extrêmes non comprises); pour 2 placettes, elle est supérieure ou égale à 1 classe.

Finalement, cette extrapolation est-elle valable, compte tenu de nos objectifs (étude des liaisons sols-production, examen de la validité de la table de production pour les peuplements sur sol hydromorphe? Remarquons que ces objectifs font appel à des études statistiques effectuées sur un grand nombre de placettes.

Nous avons donc procédé à partir des chiffres reproduits dans le tableau 1, à une comparaison de moyenne pour 2 séries appariées de faible effectif. Les moyennes ne diffèrent pas significativement (on trouve $t=1,14$, alors que pour un risque de $5 \%$ la table donne $t=2,23$.)

La méthode des sondages à la tarière est donc probablement suffisante dans le cas d'une extrapolation, pour nos placettes sur sols hydromorphes.

\subsection{Interpolation.}

Nous avons effectué le même essai que précédemment, les points retenus correspondant cette fois à la hauteur à 69 ans (âge maximum des placettes sur sol hydromorphe) au 2/3 et au $1 / 3$ de cette hauteur. HD 50 déduit de la courbe obtenue à l'aide de ces points s'avère très voisin de la valeur obtenue par analyse de tige.

\subsection{Conclusion.}

L'indice de productivité HD 50 retenu semble pouvoir être obtenu valablement par la méthode des sondages à la tarière, au moins dans le cadre des objectifs de cette étude. Par contre, des erreurs importantes sont fort possibles dans le cas d'un petit nombre de placettes; il ne faut pas utiliser cette méthode si l'on cherche à prédire avec grande précision HD 50 d'un peuplement donné, surtout s'il a nettement moins de 50 ans. 
Afin d'augmenter la précision de la méthode, il serait peut-être intéressant d'effectuer un sondage de plus. Par exemple au 1/4, à la moitié, et au 3/4 de la hauteur de l'arbre.

\section{4. - SOLS HYDROMORPHES LORRAINS ET TABLE DE PRODUCTION}

La méthode utilisée ici, comme dans d'autres études analogues (DECOURT et Le TACoN, 1970) consiste à évaluer un indice de productivité, ici HD 50, et à calculer la production probable correspondante à partir de la liaison « production totale-hauteur ». Ici, cette liaison est fournie par la table de production, et l'étude se borne à vérifier que les courbes de croissance « hauteur dominante-âge » de la table sont bien valables pour les peuplements sur sols hydromorphes.

On suppose donc, si on veut évaluer des productions, que la liaison "production totalehauteur » de la table est également valable dans le cas des sols hydromorphes. L'indépendance de cette liaison et des conditions de station (pour un même climat général) est encore discutée notamment par l'école d'Assmann (AssmanN, 1951), qui estime qu'il n'y a pas indépendance, alors que d'autres auteurs pensent que les différences constatées entre placettes ne sont pas dues aux différences de stations. Il y a sans doute un ensemble de causes variées, dont l'étude permettrait d'affiner la loi de EICHHORN. Cependant, ces différences ne portent que sur de faibles écarts de production totale, de l'ordre de $\pm 10 \%$. Dans une étude comme la nôtre, cette approximation est sans doute suffisante.

Reste à vérifier, si les courbes de croissance « hauteur-âge » de la table sont valables dans le cas particulier des sols hydromorphes. A cet effet, deux comparaisons ont été faites.

1) Comparaison de l'indice HD 50 obtenu par sondage à la tarière dans les microplacettes, à celui tiré de la table de production à partir de la hauteur dominante à l'âge de 30 ans évalué dans ces placettes (cas volontairement très défavorable).

2) Comparaison de ce même indice HD 50 à celui tiré de la table de production à partir de la hauteur dominante «actuelle » effectivement mesuré dans les microplacettes (cas d'utilisation normale d'une table de production).

Les données nécessaires à ces comparaisons figurent dans le tableau 2.

\section{1. - Validité de la table de production dans le cas où l'on ne dispose que de la hauteur dominante à 30 ans.}

Nous n'avons en fait pas comparé véritablement des hauteurs dominantes à 50 ans, mais des classes de production (déduites des valeurs de HD 50).

Pour les placettes à faible productivité, nous sommes partis de HD 35 ou même HD 40, c'est-à-dire de l'âge minima mentionné dans la table, et non de HD 30. La classe O correspond à une classe exceptionnelle, rajoutée au-dessus de la classe 1 .

La comparaison entre HD 50 réel et HD 50 tiré de la table de production à partir de HD 30 (ou HD 35 ou HD 40) fournit les résultats suivants : sur 97 placettes, la différence est inférieure ou égale à $1 / 2$ classe de production pour 67 placettes, comprise entre $1 / 2$ et 1 classe pour 22 placettes, supérieure ou égale à 1 classe pour 8 placettes.

Compte tenu des résultats concernant la valeur de la méthode des sondages à la tarière 
TABLEAU 2

Production des placettes

TABLE 2

Yield of the plots

\begin{tabular}{|c|c|c|c|c|c|c|}
\hline $\begin{array}{c}\text { No } \\
\text { Placette }\end{array}$ & $\begin{array}{l}\text { Age } \\
\text { (ans) } \\
\text { (1) }\end{array}$ & $\begin{array}{l}\text { HD } \\
(\mathrm{m}) \\
(2)\end{array}$ & $\begin{array}{c}\text { HD } 50 \\
(\mathrm{~m}) \\
(3)\end{array}$ & $\begin{array}{l}\text { Classe } \\
\text { réelle } \\
\text { (4) }\end{array}$ & $\begin{array}{c}\text { Classe tirée } \\
\text { de HD } 30 \\
\text { (5) }\end{array}$ & $\begin{array}{c}\text { Classe tirée } \\
\text { de HD } \\
(6)\end{array}$ \\
\hline 1 & 43 & 22,1 & 23,8 & 3,0 & 2,6 & 2,7 \\
\hline 2 & 44 & 21,6 & 23,8 & 3,0 & 2,6 & 3,0 \\
\hline 3 & 43 & 20,9 & 22,1 & 3,6 & 2,3 & 3,1 \\
\hline 4 & 44 & 21,4 & 24,5 & 2,8 & 2,8 & 3,0 \\
\hline 7 & 44 & 18,3 & 20,2 & 4,3 & 3,8 & 4,1 \\
\hline 8 & 50 & 15,5 & 15,5 & 5,8 & 6,0 & 5,8 \\
\hline 9 & 46 & 27,8 & 29,8 & 1,0 & 1,6 & 1,2 \\
\hline 10 & 45 & 26,4 & 28,8 & 1,4 & 1,8 & 1,6 \\
\hline 11 & 48 & 26,0 & 26,7 & 2,1 & 2,4 & 2,1 \\
\hline 13 & 40 & 24,0 & 28,5 & 1,5 & 1,5 & 1,7 \\
\hline 14 & 38 & 22,5 & 28,4 & 1,5 & 2,0 & 1,8 \\
\hline 16 & 40 & 22,4 & 28,4 & 1,5 & 2,6 & 2,1 \\
\hline 17 & 38 & 22,1 & 29,3 & 1,2 & 2,0 & 1,9 \\
\hline 18 & 36 & 22,4 & 28,5 & 1,5 & 2,0 & 1,6 \\
\hline 19 & 48 & 26,5 & 28,1 & 1,6 & 3,0 & 1,8 \\
\hline 20 & 37 & 20,4 & 25,5 & 2,5 & 2,2 & 2,3 \\
\hline 21 & 42 & 22,0 & 24,9 & 2,6 & 2,4 & 2,6 \\
\hline 23 & 36 & 20,5 & 25,8 & 2,4 & 2,0 & 2,2 \\
\hline 24 & 36 & 21,2 & 28,5 & 1,5 & 1,6 & 2,0 \\
\hline 25 & 36 & 21,2 & 26,7 & 2,1 & 2,0 & 2,0 \\
\hline 26 & 36 & 19,9 & 25,5 & 2,5 & 2,4 & 2,3 \\
\hline 27 & 36 & 18,4 & 25,3 & 2,5 & 2,5 & 2,7 \\
\hline 28 & 43 & 19,9 & 22,4 & 3,5 & 3,1 & 3,4 \\
\hline 30 & 59 & 27,3 & 23,0 & 3,4 & 2,8 & 2,8 \\
\hline 31 & 55 & 29,8 & 28,1 & 1,6 & 1,2 & 1,6 \\
\hline 33 & 67 & 33,2 & 27,5 & 1,8 & 1,8 & 1,5 \\
\hline 34 & 63 & 38,4 & 33,3 & 0 & 0,5 & 0 \\
\hline 35 & 69 & 34,2 & 26,8 & 2,0 & 3,2 & 1,3 \\
\hline 37 & 68 & 28,3 & 24,8 & 2,6 & 2,5 & 3,3 \\
\hline 137 & 68 & 31,0 & 26,9 & 2,0 & 1,2 & 2,3 \\
\hline 38 & 37 & 19,9 & 26,6 & 2,1 & 2,5 & 2,4 \\
\hline 39 & 50 & 25,7 & 26,0 & 2,4 & 1,8 & 2,4 \\
\hline 40 & 44 & 26,5 & 28,8 & 1,4 & 1,8 & 1,4 \\
\hline 41 & 63 & 28,7 & 23,7 & 3,1 & 3,0 & 2,8 \\
\hline 42 & 57 & 29,3 & 26,7 & 2,1 & 2,0 & 2,0 \\
\hline 43 & 39 & 22,4 & 25,7 & 2,4 & 2,2 & 2,0 \\
\hline 44 & 50 & 28,2 & 28,2 & 1,5 & 1,6 & 1,5 \\
\hline 45 & 50 & 28,9 & 28,9 & 1,4 & 1,3 & 1,4 \\
\hline 47 & 42 & 21,7 & 25,8 & 2,4 & 2,8 & 2,7 \\
\hline 48 & 45 & 24,3 & 27,3 & 1,8 & 2,6 & 2,3 \\
\hline 49 & 45 & 24,8 & 27,1 & 1,9 & 2,4 & 2,0 \\
\hline 50 & 39 & 22,3 & 25,5 & 2,5 & 1,8 & 2,0 \\
\hline 51 & 43 & 23,2 & 25,9 & 2,4 & 2,2 & 2,3 \\
\hline 52 & 42 & 20,9 & 24,1 & 2,9 & 2,4 & 2,9 \\
\hline
\end{tabular}

(1) Age au moment de la mesure de la hauteur dominante.

(2) Hauteur dominante au moment de la mesure.

(3) Hauteur dominante à 50 ans (méthode des carottages).

(4) Classe de production correspondant à la hauteur dominante à 50 ans.

(5) Classe tirée de la table de production à partir de la hauteur dominante à 30 ans.

(6) Classe tirée de la table de production à partir de la hauteur dominante au moment de la mesure.

(1) Age the year when dominant height was measured.

(2) Dominant height the year of the measure.

(3) Dominant height at 50 years (boring method).

(4) Yield-class corresponding to HD 50.

(5) Yield-class coming from the yield-table and corresponding to dominant height at 30 years.

(6) Yield-class coming from the yield-table and corresponding to dominant height the year of the measure. 
TABLEAU 2 (suite)

\begin{tabular}{|c|c|c|c|c|c|c|}
\hline $\begin{array}{c}\text { No } \\
\text { Placette }\end{array}$ & $\begin{array}{l}\text { Age } \\
\text { (ans) } \\
(1)\end{array}$ & $\begin{array}{l}\text { HD } \\
(\mathrm{m}) \\
(2)\end{array}$ & $\begin{array}{l}\text { HD } 50 \\
(\mathrm{~m}) \\
(3)\end{array}$ & $\begin{array}{l}\text { Classe } \\
\text { réelle } \\
(4)\end{array}$ & $\begin{array}{c}\text { Classe tirée } \\
\text { de HD } 30 \\
(5)\end{array}$ & $\begin{array}{c}\text { Classe tirée } \\
\text { de HD } \\
\text { (6) }\end{array}$ \\
\hline 53 & 41 & 21,9 & 27,1 & 1,9 & 2,8 & 2,5 \\
\hline 54 & 38 & 17,8 & 20,9 & 4,0 & 2,6 & 3,3 \\
\hline 55 & 36 & 21,5 & 28,2 & 1,5 & 1,8 & 1,8 \\
\hline 57 & 38 & 21,2 & 26,4 & 2,2 & 1,8 & 2,0 \\
\hline 58 & 46 & 20,9 & 22,8 & 3,4 & 2,9 & 3,5 \\
\hline 59 & 41 & 21,5 & 24,5 & 2,8 & 2,4 & 2,6 \\
\hline 60 & 45 & 21,8 & 23,4 & 3,2 & 2,9 & 3,0 \\
\hline 61 & 41 & 20,9 & 24,8 & 2,6 & 2,5 & 2,8 \\
\hline 67 & 36 & 17,2 & 22,9 & 3,4 & 3,2 & 3,2 \\
\hline 68 & 36 & 18,0 & 23,1 & 3,3 & 2,6 & 3,0 \\
\hline 70 & 36 & 15,3 & 25,0 & 2,6 & 3,4 & 3,8 \\
\hline 71 & 45 & 26,1 & 27,9 & 1,6 & 1,8 & 1,7 \\
\hline 72 & 42 & 22,3 & 24,4 & 2,8 & 2,4 & 2,5 \\
\hline 73 & 45 & 25,4 & 27,5 & 1,8 & 2,5 & 1,9 \\
\hline 74 & 42 & 23,0 & 25,8 & 2,4 & 2,2 & 2,2 \\
\hline 75 & 38 & 21,8 & 27,0 & 2,0 & 2,2 & 2,0 \\
\hline 76 & 38 & 23,3 & 29,5 & 1,2 & 2,0 & 1,6 \\
\hline 77 & 38 & 19,6 & 23,5 & 3,2 & 2,5 & 2,7 \\
\hline 78 & 38 & 24,9 & 29,7 & 1,1 & 1,5 & 1,1 \\
\hline 80 & 37 & 23,6 & 29,4 & 1,2 & 1,5 & 1,3 \\
\hline 81 & 37 & 22,7 & 29,1 & 1,3 & 2,0 & 1,7 \\
\hline 82 & 37 & 23,0 & 28,6 & 1,5 & 1,6 & 1,6 \\
\hline 83 & 37 & 20,1 & 25,4 & 2,5 & 2,2 & 2,4 \\
\hline 85 & 46 & 24,5 & 26,1 & 2,3 & 1,7 & 2,3 \\
\hline 86 & 57 & 29,5 & 26,2 & 2,3 & 2,8 & 1,9 \\
\hline 87 & 37 & 21,7 & 30,9 & 0,6 & 2,6 & 1,9 \\
\hline 89 & 43 & 23,7 & 27,0 & 2,0 & 2,4 & 2,2 \\
\hline 90 & 43 & 22,6 & 25,2 & 2,5 & 2,4 & 2,5 \\
\hline 91 & 43 & 21,4 & 25,2 & 2,5 & 3,2 & 2,9 \\
\hline 93 & 51 & 27,2 & 26,8 & 2,0 & 3,0 & 2,0 \\
\hline 94 & 48 & 27,2 & 28,2 & 1,5 & 2,0 & 1,6 \\
\hline 96 & 40 & 23,4 & 28,1 & 1,6 & 1,9 & 1,8 \\
\hline 97 & 58 & 33,9 & 31,5 & 0,5 & 1,0 & 0,4 \\
\hline 98 & 38 & 24,5 & 30,4 & 0,8 & 1,4 & 1,2 \\
\hline 99 & 36 & 22,9 & 28,4 & 1,5 & 1,4 & 1,4 \\
\hline 100 & 40 & 23,9 & 27,3 & 1,8 & 1,5 & 1,8 \\
\hline 102 & 58 & 34,0 & 31,6 & 0,5 & 0,6 & 0,3 \\
\hline 104 & 37 & 22,0 & 27,1 & 1,9 & 1,6 & 2,2 \\
\hline 105 & 36 & 19,7 & 25,5 & 2,5 & 2,6 & 2,4 \\
\hline 106 & 36 & 20,8 & 26,5 & 2,2 & 2,0 & 2,0 \\
\hline 107 & 61 & 32,2 & 29,0 & 1,4 & 1,3 & 1,3 \\
\hline 141 & 47 & 23,0 & 23,6 & 3,1 & 2,0 & 2,9 \\
\hline 142 & 47 & 24,1 & 24,8 & 2,6 & 1,6 & 2,6 \\
\hline 143 & 47 & 25,0 & 26,4 & 2,2 & 1,8 & 2,3 \\
\hline 144 & 47 & 25,5 & 26,3 & 2,2 & 1,6 & 2,1 \\
\hline 145 & 47 & 25,1 & 26,2 & 2,3 & 2,0 & 2,3 \\
\hline 146 & 47 & 25,4 & 26,1 & 2,3 & 1,6 & 2,1 \\
\hline 151 & 42 & 16,9 & 19,7 & 4,4 & 4,0 & 4,2 \\
\hline 152 & 54 & 21,2 & 19,5 & 4,5 & 4,4 & 4,4 \\
\hline 251 & 41 & 16,3 & 18,9 & 4,6 & 4,0 & 4,3 \\
\hline 161 & 38 & 16,2 & 19,7 & 4,4 & 3,5 & 3,8 \\
\hline 261 & 38 & 15,6 & 19,3 & 4,5 & 3,8 & 4,0 \\
\hline 147 & 56 & 18,8 & 18,1 & 4,9 & 4,7 & 5,4 \\
\hline
\end{tabular}


(chapitre 3), on peut considérer que, dans l'ensemble, la table de production s'applique probablement aux sols hydromorphes lorrains.

On remarque que parmi les 20 placettes dont la production est la plus faible, 19 ont une production inférieure à celle tirée de la table de production en partant de la hauteur dominante à 30 ans. Cela semble signifier que, pour ces placettes, un important facteur limitant (en l'occurence l'hydromorphie) se manifeste plus après 30 ans (ou 35 ou 40 ans) que ne le font, dans les placettes utilisées pour la table de production, les autres facteurs limitants. Cela se conçoit parfaitement, d'autant plus que les placettes les moins productives parmi celles utilisées pour la table de production sont situées sur calcaire assez superficiel : or on sait que c'est surtout chez les jeunes Épicéas, jusqu'à environ 10 ans, que le calcaire limite la croissance.

Le phénomène inverse se manifeste dans le cas des placettes à forte production : HD 50 est, dans la très grande majorité des cas, supérieure à ce que laisserait prévoir la table de production.

En résumé, si l'on part de HD 30, la table de production semble pouvoir s'appliquer assez valablement pour les sols hydromorphes lorrains, avec certaines modifications à apporter dans les cas extrêmes, surtout pour les sols les moins productifs.

\section{2. - Validité de la table de production dans le cas d'utilisation normale}

La différence entre HD 50 évaluée par sondage et HD 50 tiré de la table à partir de la hauteur dominante " actuelle » est inférieure ou égale à $1 / 2$ classe de production pour 87 placettes sur les 97 , comprise entre $1 / 2$ et 1 classe pour 8 placettes et supérieure à 1 classe pour 2 placettes.

La figure 1 illustre bien ces résultats. On a porté sur un même graphique les courbes de croissance en hauteur de la table de production pour les classes 1 et 2 et les courbes de croissance, construites à partir des sondages à la tarière, des arbres caractérisant les placettes classées au moment de la mesure entre les classes 1 et 2 ou au-dessus de la classe 1 (fig. 1.a). De même pour les arbres compris entre les classes 4 et 5 ou au-dessous de la classe 5 (fig. 1.b). De même également pour les 10 arbres les plus voisins de la classe moyenne, c'est-àdire de la classe 3 (fig. 1.c).

On constate, qu'au-dessus de l'âge le plus faible figurant dans les tables, les résultats sont excellents avec une légère tendance à une allure de croissance plus rapide que celle de la table pour les meilleures placettes, et moins rapide pour les moins bonnes. Les placettes moyennes sont remarquablement en accord avec la table. Ces faits confirment les observations faites au $\S 4.1$.

L'ensemble de ces résultats permet de conclure que l'utilisation de la table de production permet un classement correct selon leur indice de productivité, de la grande majorité des peuplements sur sol hydromorphe.

\section{5. - PRODUCTION DE L'ÉPICÉA SUR LES SOLS « HYDROMORPHES »}

Le tableau 3 représente la répartition des micro-placettes sur sols hydromorphes en fonction des classes de production (tirées des tables de production à partir de la hauteur dominante à 50 ans trouvée par la méthode des sondages). Les accroissements moyens 
annuels y figurent également; rappelons qu'il s'agit de la production maximum possible compte-tenu de l'écologie, les autres facteurs (sylvicoles, sanitaires, etc...) étant considérés comme se trouvant à leur optimum.

La gamme des classes de production représentées est très étendue ( 0 à 6$)$, les accroissements moyens varient de 5,2 à environ $22,5 \mathrm{~m}^{3} / \mathrm{ha} / \mathrm{an}$ à 50 ans. Néanmoins, la grande majorité des placettes sont comprises entre les classes 1 et $4(87 \%)$ et même 1 et $3(75 \%)$. Peu de placettes ont une production inférieure à celle de la classe $4(8 \%)$ ou de la classe $3(20 \%)$. Dans l'ensemble, les productions sont donc assez bonnes. Les facteurs limitants particuliers à ces sols n'affectent très fortement la production que dans les cas les plus défavorables : texture argileuse apparaissant à faible profondeur combinée à des conditions écologiques favorisant soit une nappe de printemps particulièrement durable, soit un dessèchement accentué du sol au cours de l'été.

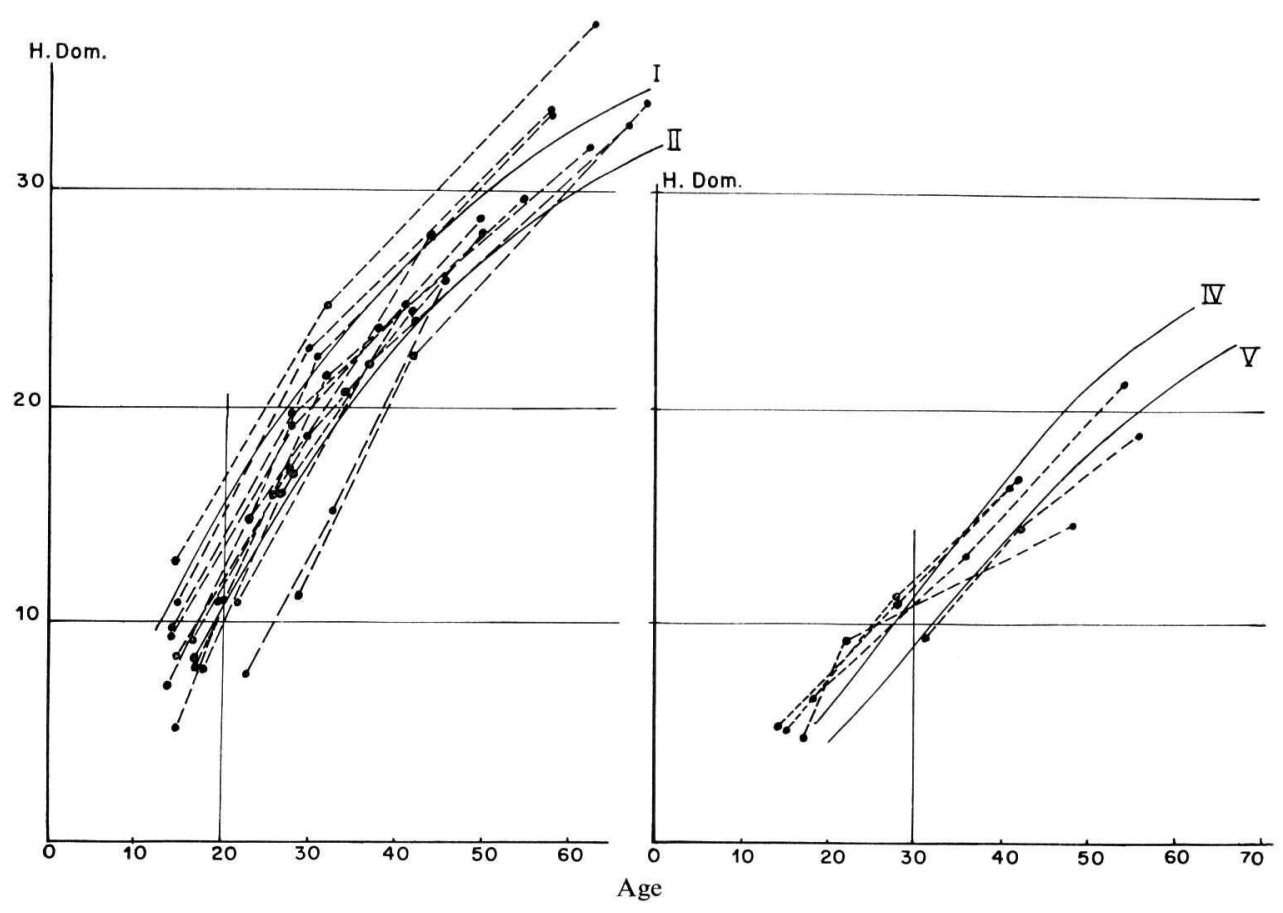

FIG. 1. - Comparaison des courbes de croissance obtenues par la méthode des sondages et de celles de la table de production (DECOURT, 1972).

1-a. Microplacettes supérieures à la classe 1 ou comprises entre les classes 1 et 2 de la table, au moment de la mesure.

1-b. Microplacettes inférieures à la classe 5 ou comprises entre les classes 4 et 5 de la table, au moment de la mesure.

FIG. 1. - Comparison between the growth curves obtained by the boring method and those corresponding to the yield-table (DECOURT, 1972).

1-a. Microplots upper than class 1 or included beetween the classes 1 and 2 of the table, the year of the measure.

1-b. Microplots lower than class 5 or included beetween the classes 4 and 5 of the table, the year of the measure. 


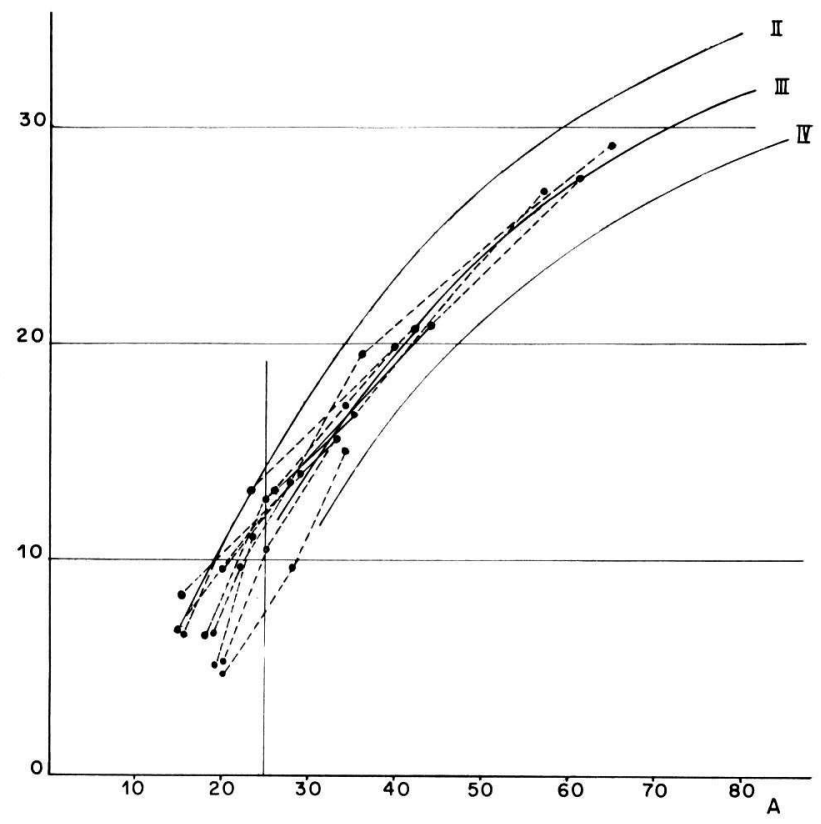

FIG. 1.

1-c. Les 10 microplacettes les plus voisines de la classe 3 de la table, au moment de la mesure.

FIG. 1.

1-c. The 10 nearest from the class 3 of the table microplots, the year of the measure.

\section{TABLEAU 3}

Répartition comparée des placettes ayant servi à construire la table de production et des microplacettes sur sols hydromorphes, par classes de productivité

\section{TABLE 3}

Compared allotment of the plots which served to realize the yield-table and the microplots on hydromorphic soils, by class of productivity

\begin{tabular}{|c|c|c|c|c|c|}
\hline \multirow{2}{*}{ Classe } & \multicolumn{2}{|c|}{$\begin{array}{l}\text { microplacettes } \\
\text { (hydromorphes) }\end{array}$} & \multicolumn{2}{|c|}{$\begin{array}{c}\text { placettes } \\
\text { (table de production N.E.) }\end{array}$} & \multirow{2}{*}{$\begin{array}{c}\text { Production } \\
\text { moyenne } \\
\text { à } 50 \text { ans } \\
\mathrm{m}^{3} / \mathrm{ha} / \mathrm{an}\end{array}$} \\
\hline & Nombre & $\%$ & Nombre & $\%$ & \\
\hline \multirow[t]{2}{*}{$\begin{array}{l}0-1 \\
1-2 \\
2-3 \\
3-4 \\
4-5 \\
5-6 \\
<6\end{array}$} & $\begin{array}{r}5 \\
31 \\
40 \\
12 \\
8 \\
1 \\
0\end{array}$ & $\begin{array}{r}5 \\
32 \\
42 \\
12 \\
8 \\
1 \\
0\end{array}$ & $\begin{array}{r}10 \\
37 \\
47 \\
25 \\
7 \\
1 \\
0\end{array}$ & $\begin{array}{r}8 \\
29 \\
37 \\
20 \\
5 \\
1 \\
0\end{array}$ & $\begin{array}{r}20,8 \\
17,8 \\
14,8 \\
11,8 \\
9,4 \\
6,2 \\
\end{array}$ \\
\hline & 97 & 100 & 127 & 100 & \\
\hline
\end{tabular}


Six placettes ont une production supérieure à celle correspondant à la classe 1 de la table. Cinq d'entre elles sont situées sur limons recouvrant les grès de Ruaux : les sols sont en fait très peu hydromorphes, très favorables à un enracinement profond; de plus la pluviosité $\mathrm{y}$ est supérieure à celle de la moyenne des placettes. La sixième possède une nappe permanente en profondeur, ce qui assure une bonne alimentation en eau pendant l'été.

Enfin, on peut constater sur le tableau 3 que la répartition par classes de productivité des microplacettes sur sols hydromorphes et de l'ensemble des placettes ayant servi à construire la table de production est tout à fait semblable, ce qui confirme les remarques faites ci-dessus.

\title{
6. - CONCLUSIONS
}

La méthode des sondages à la tarière permet une évaluation rapide de l'indice de productivité des peuplements (hauteur dominante à 50 ans) quel que soit leur âge. Cette méthode est peu précise, mais donne des résultats non biaisés et convient donc à des études de liaisons « station-production ». Cependant, sa mise en œuvre nécessite un grimpage qui n'est pas sans danger pour l'opérateur.

La table de production pour l'Épicéa commun dans le Nord-Est (DecourT, 1972), bien qu'établie à partir de peuplements situés sur des sols très variés, s'applique bien au cas particulier des peuplements sur sols hydromorphes.

Pourtant, dans les cas les plus extrêmes, les meilleures placettes ont tendance à croître plus vite, les moins bonnes plus lentement, que ne le prévoit la table.

Sur ces sols, enfin, la production de l'Épicéa est généralement excellente $(75 \%$ des peuplements dans les trois meilleures classes de productivité), sauf dans les conditions écologiques les plus défavorables ( $8 \%$ de peuplements inférieurs à la classe 4$)$.

L'interprétation plus précise des relations station-production est en cours et fera l'objet de publications ultérieures.

Reçu pour publication en mai 1974.

SUMMARY

PRODUCTIVITY OF NORWAY SPRUCE ON HYDROMORPHIC SOILS IN LORRAINE :

MEASURE AND GENERAL CHARACTERISTICS

\begin{abstract}
About hundred plots were choised for this inquest concerning adult Norway Spruce. In the concerned area annual precipitations vary from 700 to $1000 \mathrm{~mm}$. Parent materials are the most frequently formed by marls or clays of trias or lias, often covered by a more or less thick layer of loam. Soils are various : for instance brown marbled soils, pseudogleys, pelosols.

We used a method consisting in boring with Pressler's borer at various heights on dominant trees; then we reconstituted the height growth curve and, by inter- or extrapolation, obtained the dominant height at the reference age of 50 years (productivity index). This method has a low precision but is rapid and seems to be valid for a study of soil-yield relations concerning many plots.

The yield-table for Norway Spruce in the North-east (DECOurT 1972) is well suitable, roughly, for the special case of stands on hydromorphic soils. However, in the most unfavourable sites
\end{abstract}


(very important hydromorphy), that yield-table is too optimist : the noxious effect of this hydromorphy seems to be accentuated for mature trees. On the contrary, for the most productive sites, growth tends to be faster than given by the yield-table.

The mean annual volume increments, for the whole plots, vary from 5.2 to $22.5 \mathrm{~m}^{3} / \mathrm{ha} / \mathrm{year}$ at 50 years. Nevertheless only $8 \%$ of the stands are lower than class 4 and $20 \%$ than class 3 . The limiting factors of these soils strongly reduce yield only in the most unfavourable events : more or less imprevious texture at small deepness, joined with either a very durable spring water-table or a strong dryness of the soil during summer.

\section{RÉFÉRENCES BIBLIOGRAPHIQUES}

Assmann, E., 1961. - Wald Ertragskunde BLV Verlagsges., München, 1961.

Decourt N., Le Tacon F., 1970. - L'Épicéa commun sur les plateaux calcaires de l'Est de la France. Essai de prévision de la production à l'aide de déterminations pédologiques simples. Ann. Sci. forest. 27 (3).

Decourt N., 1972. - Tables de production provisoires pour l'Épicéa commun dans le N.-E. de la France. Ann. Sci. forest. 29 (1), 49-63.

Garbaye, J., Leroy Ph., Le Tacon F., Lévy G., 1970. — Réflexions sur une méthode d'étude des relations entre caractéristiques des sols et caractéristiques des peuplements. Ann. Sci. forest. 27 (3), 303-321. 